Y. Okabe

Nagoya Math. J.

Vol. 70 (1978), 81-104

\title{
INNOVATION PROCESSES ASSOCIATED WITH STATIONARY GAUSSIAN PROCESSES WITH APPLICATION TO THE PROBLEM OF PREDICTION
}

\author{
YASUNORI OKABE
}

\section{§1. Introduction}

As a continuation of the previous paper [7], we shall consider in this paper the problem of prediction given bounded intervals and obtain integral representations of predictors and prediction errors. For that purpose we shall introduce innovation processes well matched with bounded intervals. We follow the notation and terminology in [6].

Let $\boldsymbol{X}=(X(t) ; t \in \boldsymbol{R})$ be a real separable and measurable stationary Gaussian process on a probability space $(\Omega, F, P)$ with expectation zero which is continuous in the mean and purely nondeterministic. Furthermore we suppose that $\boldsymbol{X}$ has the $N$-ple Markovian property in the broad sense ([7]). We then know that the spectral measure of $\boldsymbol{X}$ has a Hardy density $\Delta$ whose outer part $h$ is expressed in the form

$$
\left\{\begin{array}{l}
h(\lambda)=\frac{Q(-\lambda)}{P(-\lambda)} \quad(\lambda \in R), \\
P(\lambda)=\sum_{n=0}^{N} c_{n}(-i \lambda)^{n}, \quad Q(\lambda)=\sum_{n=0}^{N-1} b_{n}(-i \lambda)^{n}, \quad c_{n}, b_{n} \in R, \quad c_{N} \neq 0 \\
\text { and } \\
V_{P} \subset C^{+}, \quad V_{Q} \subset C^{+} \cup \boldsymbol{R}, \quad V_{p} \cap V_{Q}=\phi,
\end{array}\right.
$$

where $V_{S}$ denotes the set of zero points of a polynomial $S$.

In [7], we have constructed an $N$-dimensional stationary Gaussian process $\mathscr{X}=(\mathscr{X}(t) ; t \in R)$ satisfying

$$
\boldsymbol{F}_{X}^{+/-}(t)=\boldsymbol{F}_{\mathscr{x}}^{+/-}(t)=\sigma(\mathscr{X}(t)) \quad(t \in \boldsymbol{R}) .
$$

Similarly we can obtain an $N$-dimensional stationary Gaussian process $\mathscr{Y}=(\mathscr{Y}(t) ; t \in R)$ satisfying

Received July 8, 1976. 


$$
\boldsymbol{F}_{\bar{X}}^{-{ }^{++}}(t)=\boldsymbol{F}_{\mathscr{y}}^{-{ }^{\prime+}}(t)=\sigma(\mathscr{Y}(t)) \quad(t \in \boldsymbol{R}) .
$$

Using these processes $\mathscr{X}$ and $\mathscr{Y}$, we shall define in $\S 2$ for any $a \in \boldsymbol{R}$ innovation processes $\boldsymbol{\nu}_{a}^{ \pm}=\left(\nu_{a}^{ \pm}(t) ; t \geq 0\right)$ which are standard $\left(\boldsymbol{F}_{a}^{ \pm}(t) ; t \geq 0\right)$ Brownian motions, where $\boldsymbol{F}_{a}^{+}(t)=\partial \boldsymbol{F}_{\boldsymbol{X}}(a) \quad(t=0), \quad \boldsymbol{F}_{\boldsymbol{X}}((a, a+t)) \quad(t>0)$ and $\boldsymbol{F}_{a}^{-}(t)=\partial \boldsymbol{F}_{\boldsymbol{X}}(a)(t=0), \boldsymbol{F}_{\boldsymbol{X}}((a-t, a))(t>0)$.

In $\S 3$ we shall obtain integral representations of the predictors $E\left(\mathscr{X}(a+T) \mid \boldsymbol{F}_{X}((a, a+T))\right)$ and $E\left(X(a+T+t) \mid \boldsymbol{F}_{X}((a, a+T))\right)$ (resp. $E\left(\mathscr{Y}(a-T) \mid \boldsymbol{F}_{X}((a-T, a))\right)$ and $\left.E\left(X(a-T-t) \mid \boldsymbol{F}_{X}((a-T, a))\right)\right)$ in terms of innovation processes $\nu_{a}^{+}$(resp. $\left.\nu_{a}^{-}\right)(a \in R, t>0, T>0)$. As an application of these results, we shall find that Gaussian processes $\boldsymbol{Y}_{ \pm}=$ $\left(Y_{ \pm}(t) ; t \geq 0\right)=\left(X( \pm t)-E\left(X( \pm t) \mid \partial F_{X}(0)\right) ; t \geq 0\right)$ have canonical representations ([3]).

We shall obtain in $\S 4$ integral representations of the predictors $E\left(\mathscr{X}(a-t) \mid \boldsymbol{F}_{X}((a, a+T))\right)$ and $E\left(X(a-t) \mid \boldsymbol{F}_{X}((a, a+T))\right)$ (resp. $E(\mathscr{Y}(a+$ $\left.t) \mid \boldsymbol{F}_{X}((a-T, a))\right)$ and $\left.E\left(X(a+t) \mid \boldsymbol{F}_{X}((a-T, a))\right)\right)$ in terms of innovation processes $\boldsymbol{\nu}_{a}^{+}$(resp. $\left.\boldsymbol{\nu}_{a}^{-}\right)(a \in \boldsymbol{R}, t>0, T>0)$. Representation kernels in representation theorems in $\S 3$ and $\S 4$ can be written using the solution of matrix Riccati equation.

In $\S 5$ we shall prove orthogonal decomposition theorems of integral representations of the predictors $E\left(\mathscr{X}(-a-t) \mid \boldsymbol{F}_{X}((-a, a))\right)$ and $E(X(-a$ $\left.-t) \mid \boldsymbol{F}_{X}((-a, a))\right)\left(\operatorname{resp} . E\left(\mathscr{Y}(a+t) \mid \boldsymbol{F}_{X}((-a, a))\right)\right.$ and $\left.E\left(X(a+t) \mid \boldsymbol{F}_{X}((-a, a))\right)\right)$ in terms of innovation processes $\nu_{0}^{-}$and $\nu_{-a}^{+}$(resp. $\nu_{0}^{+}$and $\left.\nu_{a}^{-}\right)(a>0$, $t>0$ ).

In $\S 6$ we shall give concrete computations in the space $Z_{4}$ of representation kernels in representation theorems in $\S 3$ and $\S 4$ and then obtain explicit representations of prediction errors of $E\left(X(a+t) \mid \boldsymbol{F}_{X}((a\right.$ $-T, a))), \quad E\left(X(a-t) \mid \boldsymbol{F}_{X}((a, a+T))\right)(a \in R, t>0, T>0)$ and $E(X( \pm(a$ $\left.+t)) \mid \boldsymbol{F}_{X}((-a, a))\right)(a>0, t>0)$.

Using the results of the previous section, we shall obtain in $\S 7$ integral representations of the predictors $E\left(\mathscr{X}(a+t) \mid \boldsymbol{F}_{X}((a-T, a))\right)$ (resp. $E\left(\mathscr{Y}(a-t) \mid \boldsymbol{F}_{X}((a, a+T))\right)$ ) in terms of innovation processes $\nu_{a}^{-}$ (resp. $\left.\nu_{a}^{+}\right)(a \in R, t>0, T>0)$ and then the predictors $E\left(\mathscr{X}(a+t) \mid \boldsymbol{F}_{X}((-a, a))\right)$ (resp. $\left.E\left(\mathscr{Y}(-a-t) \mid \boldsymbol{F}_{X}((-a, a))\right)\right)$ in terms of innovation processes $\nu_{0}^{+}$and $\nu_{a}^{-}$ (resp. $\nu_{0}^{-}$and $\left.\nu_{-a}^{+}\right)(a>0, t>0)$.

\section{§2. Innovation processes $\nu_{a}^{+}$and $\nu_{a}^{-}(a \in R)$}

We denote by $E$ the Fourier transform of $h$ in (1.1). Then we have the following canonical representation: 


$$
X(t)=\sqrt{2 \pi}^{-1} \int_{-\infty}^{t} E(t-s) d B(s),
$$

where $(B(t) ; t \in \boldsymbol{R})$ is a standard Brownian motion satisfying

$$
\boldsymbol{F}_{\bar{X}}(t)=\sigma\left(B\left(s_{1}\right)-B\left(s_{2}\right) ; s_{1}, s_{2}<t\right) \quad(t \in R) .
$$

We define an $N \times N$-matrix $A$ and an $N \times 1$-vector $\boldsymbol{b}$ by

$$
A=\left(\begin{array}{cccccc}
0 & & & & a_{0} \\
-1 & 0 & & & & a_{1} \\
-1 & 0 & & & a_{2} \\
& \ddots & \ddots & & \vdots \\
0 & & & \ddots & 0 & a_{N-2} \\
& & & & -1 & a_{N-1}
\end{array}\right) \quad \text { and } \quad \boldsymbol{b}=\left(\begin{array}{c}
b_{0} \\
b_{1} \\
\vdots \\
b_{N-1}
\end{array}\right)
$$

where $a_{n}=c_{n} c_{N}^{-1}(0 \leq n \leq N-1)$. Since the characteristic equation of $A$ is equal to $(-1)^{N} c_{N}^{-1} P\left(i^{-1} \lambda\right)$ and so all eigenvalues of $A$ have negative real parts by (1.1), we can define $N$ real $L^{2}$-functions $E_{n}(0 \leq n \leq N-1)$ and a real $L^{2}$-function $F$ by

$$
E_{n}(t)=\sqrt{2 \pi}^{-1} \chi_{(0, \infty)}(t)\left(e^{t A} \cdot \boldsymbol{b}\right)_{n} \quad(t \in \boldsymbol{R})
$$

and

$$
F(t)=-\sqrt{2 \pi} c_{N}^{-1} \chi_{(0, \infty)}(t)\left(e^{t A}\left(\begin{array}{c}
1 \\
0 \\
\vdots \\
0
\end{array}\right)\right)_{N-1} \quad(t \in R) .
$$

Using these $L^{2}$-functions $E_{n}$, we define an $N$-dimensional stationary Gaussian process $\mathscr{X}=(\mathscr{X}(t) ; t \in \boldsymbol{R})=\left(\left(X_{0}(t), \cdots, X_{N_{-1}}(t)\right)^{*} ; t \in \boldsymbol{R}\right)$ by

$$
X_{n}(t)=\sqrt{2 \pi}-1 \int_{-\infty}^{t} E_{n}(t-s) d B(s) \quad(0 \leq n \leq N-1, t \in \boldsymbol{R}) .
$$

Then we see from the results in [7] that

THEOREM 2.1 ( i ) $X_{N-1}(t)=(-2 \pi)^{-1} c_{N} X(t)(t \in R)$.

( ii ) $\left\{X_{n}(t) ; 0 \leq n \leq N-1\right\}$ is linearly independent in $\boldsymbol{M}_{\boldsymbol{X}}$ for any $t \in \boldsymbol{R}$.

(iii ) $\boldsymbol{M}_{\bar{X}}^{-}(t)=\boldsymbol{M}_{\dot{x}}^{-}(t)$ and $\boldsymbol{F}_{\bar{X}}^{-}(t)=\boldsymbol{F}_{\dot{x}}^{-}(t)(t \in \boldsymbol{R})$.

(iv ) $\boldsymbol{M}_{\boldsymbol{X}}^{+/-}(t)$ is equal to the linear hull of $\left\{X_{n}(t) ; 0 \leq n \leq N-1\right\}(t \in \boldsymbol{R})$.

( v ) $\quad \boldsymbol{F}_{X}^{+1-}(t)=\boldsymbol{F}_{\mathscr{x}}^{+/-}(t)=\sigma(\mathscr{X}(t))(t \in \boldsymbol{R})$. 


$$
\begin{aligned}
& \text { (vi ) } \mathscr{X}(t)-\mathscr{X}(s)=\sqrt{2 \pi^{-1}}(B(t)-B(s)) \boldsymbol{b}+\int_{s}^{t} A \mathscr{X}(u) d u(s<t) . \\
& \text { (vii) } \mathscr{X}(t)=e^{(t-s) A} \mathscr{X}(s)+\sqrt{2 \pi^{-1}} \int_{s}^{t} e^{(t-u) A} \cdot \boldsymbol{b} d B(u)(s<t) . \\
& \text { (viii) } E\left(X(t) \mid \boldsymbol{F}_{\bar{X}}^{-}(s)\right)=\sum_{n=0}^{N-1}(-1)^{n} \boldsymbol{F}^{(n)}(t-s) X_{n}(s)(s<t) . \\
& \text { (ix) } E\left(\mathscr{X}(t) \mid \boldsymbol{F}_{\mathscr{x}}^{-}(s)\right)=e^{(t-s) A} \mathscr{X}(s)(s<t) .
\end{aligned}
$$

[2.1] Now we fix any $a \in \boldsymbol{R}$ and define the $\sigma$-fields $\boldsymbol{F}_{a}^{+}(t)(t \geq 0)$ by

$$
\boldsymbol{F}_{a}^{+}(t)= \begin{cases}\partial \boldsymbol{F}_{X}(a) & (t=0), \\ \boldsymbol{F}_{X}((a, a+t)) & (t>0) .\end{cases}
$$

Then we shall show

THEOREM 2.2. There exists a standard Brownian motion $\nu_{a}^{+}=$ $\left(\nu_{a}^{+}(t) ; t \geq 0\right)$ such that

(i ) $\nu_{a}^{+}(0)=0$,

(ii) $\boldsymbol{F}_{a}^{+}(t)=\partial \boldsymbol{F}_{X}(\alpha) \vee \sigma\left(\nu_{a}^{+}(s) ; 0 \leq s \leq t\right)(t \geq 0)$,

(iii) $\nu_{a}^{+}$is independent. of the $\sigma$-field $\partial F_{X}(a)$,

(iv) $X_{n_{0}}(t+a)-X_{n_{0}}(a)=\sqrt{2 \pi}^{-1} b_{n_{0}} \nu_{a}^{+}(t)+b_{n_{0}} \boldsymbol{e} \cdot \int_{a}^{a+t} E\left(\mathscr{X}(s) \mid \boldsymbol{F}_{X}((a, s))\right) d s$ $(t \geq 0)$,

where $n_{0}=\max \left\{n \in\{0,1, \cdots, N-1\} ; b_{n} \neq 0\right\}$ and $b_{n_{0}} e=$ the $n_{0}+1$-th row of the matrix $A$.

Proof. By (iv) we define a stochastic process $\nu_{a}^{+}=\left(\nu_{a}^{+}(t) ; t \geq 0\right)$ with continuous paths. It then follows from (2.2), Theorem 2.1 (iii) and (iv) that $\nu_{a}^{+}$is a square integrable $\left(\boldsymbol{F}_{a}^{+}(t) ; t \geq 0\right)$-martingale with expectation zero. We put $M=N-1-n_{0}$. It is easy to see from Lemma 2.4, Theorem 2.1 (i) and Lemma 4.1 (i) in [7] that $E^{(n)}(0+)=0(0 \leq n \leq M-1)$. This implies by (2.1) that $X(t)$ is $M$-times differentiable in the mean and a stationary Gaussian process $X^{(M)}=\left(X^{(M)}(t) ; t \in R\right)$ has the same property as $X$. Applying Theorem 2.1 in this paper to the process $X^{(M)}$, we have an $N$-dimensional stationary Gaussian process $\mathscr{X}_{M}=\left(\mathscr{X}_{M}(t) ; t \in \boldsymbol{R}\right)$ such that $\mathscr{X}_{M, N-1}(t)=(-2 \pi)^{-1} c_{N} X^{(M)}(t)$ and

$$
\begin{aligned}
& \mathscr{X}_{M, N-1}(t+a)-\mathscr{X}_{M, N-1}(a) \\
& \quad=(-1)^{M} b_{n_{0}} \sqrt{2 \pi^{-1}}(B(t+a)-B(a))+\int_{a}^{a+t}\left(A \mathscr{X}_{M}(u)\right)_{N-1} d u \quad(t \geq 0) .
\end{aligned}
$$

Using this process $\mathscr{X}_{M}$, we define an $\boldsymbol{R}^{N}$-valued stochastic process 
$(\mathscr{Z}(t) ; t \geq 0)$ and a real valued stochastic process $(Y(t) ; t \geq 0)$ by

$$
\left\{\begin{array}{l}
\mathscr{Z}(t)=\mathscr{X}_{M}(t+a)-E\left(\mathscr{X}_{M}(t+a) \mid \partial \boldsymbol{F}_{X}(a)\right), \\
Y(t)=(-1)^{M} \sqrt{2 \pi} \boldsymbol{e} \cdot \int_{0}^{t} \mathscr{Z}(s) d s+B(t+a)-B(a),
\end{array}\right.
$$

and then a real valued stochastic process $\left(\eta_{a}^{+}(t) ; t \geq 0\right)$ by

$$
\eta_{a}^{+}(t)=Y(t)-(-1)^{M} \sqrt{2 \pi} \boldsymbol{e} \int_{0}^{t} E\left(\mathscr{Z}(s) \mid \boldsymbol{F}_{X}((a, a+s))\right) d s .
$$

Then it follows from the results of [4] and [5] that $\left(\eta_{a}^{+}(t) ; t \geq 0\right)$ is a standard $\left(\boldsymbol{F}_{a}^{+}(t) ; t \geq 0\right)$-Brownian motion for which $\sigma\left(\eta_{a}^{+}(s) ; 0 \leq s \leq t\right)$ is equal to $\sigma(Y(s) ; 0 \leq s \leq t)$ and $\sigma\left(\eta_{a}^{+}(u)-\eta_{a}^{+}(v) ; u, v>t\right)$ is independent of $\boldsymbol{F}_{a}^{+}(t)$. By the definition of the process $(Y(t) ; t \geq 0), \quad Y(t)=$ $(-1)^{M} b_{n_{0}}^{-1}\left(\mathscr{X}_{M, N-1}(t+a)-E\left(\mathscr{X}_{M, N-1}(t+a) \mid \partial \boldsymbol{F}_{X}(a)\right)\right)$. Therefore, noting that $\partial F_{X}(a)=\sigma\left(X^{(n)}(a) ; 0 \leq n \leq M\right)$ and $\mathscr{X}_{M, N-1}(t)=(-2 \pi)^{-1} c_{N} X^{(M)}(t)$, we find that $\sigma(Y(s) ; 0 \leq s \leq t) \vee \partial \boldsymbol{F}_{X}(a)=\boldsymbol{F}_{X}((a, a+t))$ and so $\boldsymbol{F}_{a}^{+}(t)=\partial \boldsymbol{F}_{X}(a) \vee \sigma\left(\eta_{a}^{+}(s) ;\right.$ $0 \leq s \leq t)$. On the other hand, by the definition of the processes $\left(\nu_{a}^{+}(t)\right.$; $t \geq 0)$ and $\left(\eta_{a}^{+}(t) ; t \geq 0\right)$, we see that $\left(\nu_{a}^{+}(t)-\eta_{a}^{+}(t) ; t \geq 0\right)$ is a bounded variation process. Since $\left(\nu_{a}^{+}(t) ; t \geq 0\right)$ and $\left(\eta_{a}^{+}(t) ; t \geq 0\right)$ are continuous $\left(\boldsymbol{F}_{a}^{+}(t)\right.$; $t \geq 0)$-martingales, we find that $\nu_{a}^{+}(t)=\eta_{a}^{+}(t)$ for any $t \in[0, \infty)$ and so this completes the proof of Theorem 2.2.

(Q.E.D.)

[2.2] Next we define the $\sigma$-fields $\boldsymbol{F}_{a}^{-}(t)(t \geq 0)$ by

$$
\boldsymbol{F}_{a}^{-}(t)= \begin{cases}\partial \boldsymbol{F}_{X}(a) & (t=0) \\ \boldsymbol{F}_{\boldsymbol{X}}((a-t, a)) & (t>0)\end{cases}
$$

Noting that $\widehat{h}=\check{E}$, we see that there exists a standard Brownian motion $\left(B_{-}(t) ; t \in \boldsymbol{R}\right)$ for which the followings hold:

$$
\begin{gathered}
X(t)=\sqrt{2 \pi^{-1}} \int_{t}^{\infty} E(s-t) d B_{-}(s), \\
\boldsymbol{F}_{X}^{+}(t)=\sigma\left(B_{-}\left(s_{1}\right)-B_{-}\left(s_{2}\right) ; s_{1}, s_{2}>t\right) \quad(t \in \boldsymbol{R}) .
\end{gathered}
$$

Using this Brownian motion $\left(B_{-}(t) ; t \in R\right)$ and $N$ real $L^{2}$-functions $E_{n}$ $(0 \leq n \leq N-1)$ in (2.4), we define an $N$-dimensional stationary Gaussian process $\mathscr{Y}=(\mathscr{Y}(t) ; t \in \boldsymbol{R})=\left(\left(Y_{0}(t), \cdots, Y_{N-1}(t)\right)^{*} ; t \in \boldsymbol{R}\right)$ by

$$
Y_{n}(t)=\sqrt{2 \pi} \bar{\pi}^{-1} \int_{t}^{\infty} E_{n}(s-t) B_{-}(s) \quad(0 \leq n \leq N-1, t \in R) .
$$

Similarly as in Theorem 2.1, we have 
THEOREM 2.3. ( i ) $Y_{N-1}(t)=X_{N-1}(t)=(-2 \pi)^{-1} c_{N} X(t) \quad(t \in \boldsymbol{R})$.

(ii ) $\left\{Y_{n}(t) ; 0 \leq n \leq N-1\right\}$ is linearly independent in $\boldsymbol{M}_{\boldsymbol{X}}$ for any $t \in \boldsymbol{R}$.

(iii) $\boldsymbol{M}_{X}^{+}(t)=\boldsymbol{M}_{\dot{y}}^{+}(t)$ and $\boldsymbol{F}_{X}^{+}(t)=\boldsymbol{F}_{y}^{+}(t)(t \in \boldsymbol{R})$. $(t \in R)$.

(iv) $M_{\bar{X}}^{-1+}(t)$ is equal to the linear hull of $\left\{Y_{n}(t) ; 0 \leq n \leq N-1\right\}$

( v ) $\quad \boldsymbol{F}_{X}^{-1+}(t)=\boldsymbol{F}_{\mathscr{y}}^{-1+}(t)=\sigma(\mathscr{Y}(t))(t \in R)$.

(vi ) $\mathscr{Y}(s)-\mathscr{Y}(t)=\sqrt{2 \pi^{-1}}\left(B_{-}(t)-B_{-}(s)\right) b+\int_{s}^{t} A \mathscr{Y}(u) d u(s<t)$.

(vii) $\mathscr{Y}(s)=e^{(t-s) A} \mathscr{Y}(t)+\sqrt{2 \pi^{-1}} \int_{s}^{t} e^{(u-s) A} \cdot \boldsymbol{b} d B_{-}(u)(s<t)$.

(viii) $E\left(X(s) \mid F_{X}^{+}(t)\right)=\sum_{n=0}^{N-1}(-1)^{n} F^{(n)}(t-s) Y_{n}(t)(s<t)$.

(ix ) $E\left(\mathscr{Y}(s) \mid F_{\mathscr{Q}}^{+}(t)\right)=e^{(t-s) A \mathscr{Y}(t)}(s<t)$.

By virtue of Theorem 2.3, in the same way as Theorem 2.2, we obtain

THEOREM 2.4. There exists a standard Brownian motion $\nu_{a}^{-}=\left(\nu_{a}^{-}(t)\right.$; $t \geq 0)$ such that

(i) $\nu_{a}^{-}(0)=0$,

(ii) $\boldsymbol{F}_{a}^{-}(t)=\partial \boldsymbol{F}_{X}(a) \vee \sigma\left(\nu_{a}^{-}(s) ; 0 \leq s \leq t\right)(t \geq 0)$,

(iii) $\nu_{a}^{-}$is independent of the $\sigma$-field $\partial \boldsymbol{F}_{X}(a)$,

(iv) $Y_{n_{0}}(a-t)-Y_{n_{0}}(a)=\sqrt{2 \pi^{-1}} b_{n_{0}} \nu_{a}^{-}(t)+b_{n_{0}} e \cdot \int_{a-t}^{a} E\left(\mathscr{Y}(s) \mid F_{X}((s, a))\right) d s$ $(t \geq 0)$.

DEFINITION 2.1. We call the standard Brownian motions $\nu_{a}^{+}$(resp. $\left.\nu_{a}^{-}\right)\left(F_{a}^{+}(t) ; t \geq 0\right)-\left(\right.$ resp. $\left(F_{a}^{-}(t) ; t \geq 0\right)$ ) innovation processes associated with the stationary Gaussian process $\boldsymbol{X}$.

[2.3] Finally in this subsection we shall give a relation between the family of innovation processes $\nu_{a}^{ \pm}(a \in R)$. We have the unitary transformation group $(U(t) ; t \in R)$ acting on the space $\boldsymbol{M}_{\boldsymbol{X}}$ defined by

$$
U(t) X(s)=X(t+s) \quad(t, s \in R) .
$$

Then we shall show

THEOREM 2.5. $U(t) \nu_{a}^{ \pm}=\nu_{a+t}^{ \pm}$for any $a \in \boldsymbol{R}$ and $t \in \boldsymbol{R}$.

Proof. It is easy to see that $U(t) M_{X}((a, b))=M_{X}((a+t, b+t))$, $U(t) \partial M_{X}(a)=\partial M_{X}(a+t)$ and $U(t) M_{X}^{+/-}(a)=M_{X}^{+/-}(a+t)$. We define an $N-$ dimensional stationary Gaussian process $\mathscr{Z}(t)=\left(U(t) X_{0}(0), \cdots, U(t) X_{N-1}(0)\right)^{*}$ 
$(t \in R)$. Then it follows from Theorem 2.1 (ii) and (iv) that $\mathscr{Z}(t)$ is continuous in the mean, each component of $\mathscr{Z}(t)$ belongs to the space $\boldsymbol{M}_{\boldsymbol{X}}^{+/-}(t)$ and $\left\{U(t) X_{n}(0) ; 0 \leq n \leq N-1\right\}$ is linearly independent in $\boldsymbol{M}_{\boldsymbol{X}}$ for any $t \in R$. Therefore we see from Theorem 5.1 in [7] that there uniquely exists a constant $N \times N$-matrix $\tilde{T}$ for which $\mathscr{Z}(t)=\tilde{T} \mathscr{X}(t)$ $(t \in R)$. Since $\mathscr{Z}(0)=\mathscr{X}(0)$, we find that $\tilde{T}$ is the unit matrix and so $\mathscr{Z}(t)=\mathscr{X}(t)$. By Theorem 2.2 (iv) this implies that $U(t) \nu_{a}^{+}(s)=\nu_{a+t}^{+}(s)$. Similarly, we have $U(t) \nu_{a}^{-}(s)=\nu_{a+t}^{-}(s)$.

(Q.E.D.)

\section{§3. Integral representations of the predictors (I)}

[3.1] In this subsection we shall obtain integral representations of the predictors $E\left(\mathscr{X}(a+T) \mid \boldsymbol{F}_{a}^{+}(T)\right)(a \in \boldsymbol{R}, T \geq 0)$. For any $a \in \boldsymbol{R}$ we define $N \times N$-matrices $P_{a}(t)(t \geq 0)$ by

$$
\begin{aligned}
P_{a}(t)= & E\left\{\left(\mathscr{X}(a+t)-E\left(\mathscr{X}(a+t) \mid \boldsymbol{F}_{a}^{+}(t)\right) \cdot(\mathscr{X}(a+t)\right.\right. \\
& \left.\left.-E\left(\mathscr{X}(a+t) \mid \boldsymbol{F}_{a}^{+}(t)\right)\right)^{*}\right\}
\end{aligned}
$$

and then $N \times 1$-vectors $f_{a}(t, s)(0 \leq s \leq t<\infty)$ by

$$
\boldsymbol{f}_{a}(t, s)=e^{(t-s) A} \cdot\left(P_{a}(s) \boldsymbol{e}^{*}+\sqrt{2 \pi^{-1}} \boldsymbol{b}\right) .
$$

At first we shall show

LEMMA 3.1. $f_{a}(t, s)=(\partial / \partial s) E\left(\nu_{a}^{+}(s) \cdot \mathscr{X}(a+t)\right)(0 \leq s \leq t<\infty)$.

Proof. We put $\widetilde{\mathscr{X}}(s)=\mathscr{X}(a+s)-E\left(\mathscr{X}(a+s) \mid F_{a}^{+}(s)\right)$. It then follows from Theorems 2.1 (vi) and 2.2 (iv) that

$$
\nu_{a}^{+}(t)=e \cdot \int_{0}^{t} \tilde{\mathscr{X}}(s) d s+B(a+t)-B(a) \quad(t \geq 0) .
$$

Therefore we see from (2.2) and Theorem 2.1 (vii) that

$$
\begin{aligned}
E\left(\nu_{a}^{+}(s) \cdot \mathscr{X}(a+t)\right)= & \int_{0}^{s} E\left(\mathscr{X}(a+t) \cdot \tilde{\mathscr{X}}(\iota)^{*}\right) \boldsymbol{e}^{*} \cdot d \iota \\
& +E((B(a+s)-B(a)) \cdot \mathscr{X}(a+t)) \\
= & \int_{0}^{s} e^{(t-\iota) A} E\left(\mathscr{X}(a+\tau) \cdot \tilde{\mathscr{X}}(\iota)^{*}\right) \boldsymbol{e}^{*} d \iota \\
& +\sqrt{2 \pi^{-1}} \int_{a}^{a+s} e^{(a+t-\iota) A} \boldsymbol{b} d \iota \\
= & \int_{0}^{s} e^{(t-\iota) A}\left(P_{a}(\iota) \boldsymbol{e}^{*}+\sqrt{2 \pi^{-1}} \boldsymbol{b}\right) d \iota .
\end{aligned}
$$


On the other hand, by Corollaries 2.1 and 2.2 in [6] and the results in [7], we find that $P_{a}(\iota)$ is continuous in $\iota$ and so this implies Lemma 3.1 .

(Q.E.D.)

Lemma 3.2. For any $a \in R$ and any $T \in(0, \infty)$,

$$
E\left(\mathscr{X}(a+T) \mid F_{X}((a, a+T))\right)=E\left(\mathscr{X}(a+T) \mid \partial F_{X}(a)\right)+\int_{0}^{T} f_{a}(T, s) d \nu_{a}^{+}(s) .
$$

Proof. We put $\boldsymbol{Y}=\mathscr{X}(a+T)-E\left(\mathscr{X}(a+T) \mid \partial F_{X}(a)\right)-\int_{0}^{T} f_{a}(T, s) d \nu_{a}^{+}(s)$. It then follows from Theorem 2.2 (i), (iii) and Lemma 3.1 that $(d / d s) E\left(\boldsymbol{Y} \cdot \nu_{a}^{+}(s)\right)=0$ and so $E\left(\boldsymbol{Y} \cdot \nu_{a}^{+}(s)\right)=E\left(\boldsymbol{Y} \cdot \nu_{a}^{+}(0)\right)=0$ for any $s \in[0, T]$. Since $\boldsymbol{Y}$ is orthogonal to the space $\partial M_{X}(a)$, we see that $\boldsymbol{Y}$ is orthogonal to the closed linear hull of $\left\{\nu_{a}^{+}(s) ; 0 \leq s \leq T\right\} \cup \partial M_{X}(a)$ and so $\boldsymbol{Y}$ is independent of the $\sigma$-field generated by $\left\{\nu_{a}^{+}(s) ; 0 \leq s \leq T\right\} \cup \partial M_{X}(a)$. Therefore, by virtue of Theorem 2.2 (ii), we find that $E\left(\boldsymbol{Y} \mid \boldsymbol{F}_{X}((a, a+T))\right)=0$ and this implies Lemma 3.2.

Next we shall derive a differential equation which $P_{a}(t)$ satisfies.

LEMmA 3.3. For any $a \in R, P_{a}(t)$ is the unique solution of the following matrix Riccati equation:

$$
\left\{\begin{array}{lr}
\frac{d P_{a}(t)}{d t}=\left(A-\sqrt{2 \pi^{-1}} \boldsymbol{b} \cdot \boldsymbol{e}\right) \cdot P_{a}(t)+P_{a}(t)\left(A-\sqrt{2 \pi^{-1}} \boldsymbol{b} \cdot \boldsymbol{e}\right)^{*}-P_{a}(t) \boldsymbol{e}^{*} \cdot \boldsymbol{e} P_{a}(t) & (t>0), \\
P_{a}(0)=K_{x}(0)-\Sigma_{a}(0), &
\end{array}\right.
$$

where $\Sigma_{a}(0)=E\left\{E\left(\mathscr{X}(a) \mid \partial F_{X}(a)\right) \cdot E\left(\mathscr{X}(a) \mid \partial F_{X}(a)\right)^{*}\right\}$.

Proof. We put $\Sigma_{a}(t)=E\left\{E\left(\mathscr{X}(a+t) \mid \boldsymbol{F}_{a}^{+}(t)\right) \cdot E\left(\mathscr{X}(a+t) \mid \boldsymbol{F}_{a}^{+}(t)\right)^{*}\right\}$. Then it follows from Theorems 2.1 (xi), 2.2 (iii) and Lemma 3.2 that

$$
\Sigma_{a}(t)=e^{t A} \Sigma_{a}(0) e^{t A *}+\int_{0}^{t} f_{a}(t, s) f_{a}^{*}(t, s) d s .
$$

Noting that $P_{a}(t)=K_{x}(0)-\Sigma_{a}(t)$, we see from Lemma 5.2 in [7] that

$$
\begin{aligned}
\frac{d P_{a}(t)}{d t}= & -A \cdot \Sigma_{a}(t)-\Sigma_{a}(t) A^{*}-P_{a}(t) \boldsymbol{e}^{*} \cdot \boldsymbol{e} \cdot P_{a}(t) \\
& -\sqrt{2 \pi^{-1}} P_{a}(t) \boldsymbol{e}^{*} \cdot \boldsymbol{b}^{*}-\sqrt{2 \pi}-1 \boldsymbol{b} \cdot \boldsymbol{e} \cdot P_{a}(t)-(2 \pi)^{-1} \boldsymbol{b} \cdot \boldsymbol{b}^{*} \\
= & A \cdot\left(K_{x}(0)-\Sigma_{a}(t)\right)+\left(K_{x}(0)-\Sigma_{a}(t)\right) A^{*} \\
& -P_{a}(t) \cdot \boldsymbol{e}^{*} \cdot \boldsymbol{e} \cdot P_{a}(t)-\sqrt{2 \pi^{-1}} P_{a}(t) \boldsymbol{e}^{*} \cdot \boldsymbol{b}^{*}-\sqrt{2 \pi^{-1}} \boldsymbol{b} \cdot \boldsymbol{e} \cdot P_{a}(t)
\end{aligned}
$$




$$
\begin{aligned}
& =A \cdot P_{a}(t)+P_{a}(t) A^{*}-P_{a}(t) e^{*} \cdot e \cdot P_{a}(t) \\
& -\sqrt{2 \pi}{ }^{-1} P_{a}(t) \boldsymbol{e}^{*} \cdot \boldsymbol{b}^{*}-\sqrt{2 \pi} \overline{2}^{-1} \boldsymbol{b} \cdot \boldsymbol{e} \cdot \boldsymbol{P}_{a}(t) \\
& =\left(A-\sqrt{2 \pi^{-1}} \boldsymbol{b} \cdot \boldsymbol{e}\right) P_{a}(t)+P_{a}(t)\left(A-\sqrt{2 \pi} \overline{1}^{-1} \boldsymbol{b} \cdot \boldsymbol{e}\right)^{*}-P_{a}(t) \boldsymbol{e}^{*} \cdot \boldsymbol{e} \cdot P_{a}(t) \text {. }
\end{aligned}
$$

We define an $N \times\left(N-n_{0}\right)$-matrix $J$ by

$$
J=\left(K_{x}(0)_{\substack{m n \\ n}}\right)_{\substack{0 \leq m \leq N-1 \\ n_{0} \leq n \leq N-1}} \cdot\left(K_{x}(0)_{m n}\right)_{n_{0} \leq m, n \leq N-1}^{-1} .
$$

Then we shall show

LEMMA 3.4. $\quad \Sigma_{a}(0)=J \cdot\left(K_{\mathscr{x}}(0)_{m n}\right)_{\substack{n_{0} \leq m \leq N-1 \\ 0 \leq n \leq N-1}}=J \cdot\left(K_{\mathscr{x}}(0)_{m n}\right)_{n_{0} \leq m, n \leq N-1} \cdot J^{*}$.

In particular, $\Sigma_{a}(0)$ is independent of $a$.

Proof. Since the dimension of the space $\partial M_{X}(a)$ is $N-n_{0}$ ([1]), it follows from Theorem 2.1 (i) (ii) (vi) that $\partial M_{X}(a)$ equals the linear hull of $\left\{X_{n}(a) ; n_{0} \leq n \leq N-1\right\}$. Therefore there uniquely exists an $N \times(N$ - $\left.n_{0}\right)$-matrix $J(a)$ such that $E\left(\mathscr{X}(a) \mid \partial F_{X}(a)\right)=J(a)\left(X_{n_{0}}(a) \cdots X_{N-1}(a)\right)^{*}$. This implies that

$J(a)$

$$
\begin{aligned}
& =E\left\{\mathscr{X}(a) \cdot\left(X_{n_{0}}(a) \cdots X_{N-1}(a)\right)\right\} \cdot E\left\{\left(X_{n_{0}}(a) \cdots X_{N-1}(a)\right)^{*} \cdot\left(X_{n_{0}}(a) \cdots X_{N-1}(a)\right)\right\}^{-1} \\
& =E\left\{\mathscr{X}(0) \cdot\left(X_{n_{0}}(0) \cdots X_{N-1}(0)\right)\right\} \cdot E\left\{\left(X_{n_{0}}(0) \cdots X_{N-1}(0)\right)^{*} \cdot\left(X_{n_{0}}(0) \cdots X_{N-1}(0)\right)\right\}^{-1}
\end{aligned}
$$

and so we have Lemma 3.4 .

By the uniqueness of local solutions, we see from Lemmas 3.3 and 3.4 that

LEMMA 3.5. For any $a, b \in R P_{a}(t)=P_{b}(t)(t \geq 0)$.

Consequently, combining above lemmas, we obtain

THEOREM 3.1. For any $a \in R$ and any $T \in(0, \infty)$,

$$
\begin{aligned}
& E\left(\mathscr{X}(a+T) \mid \boldsymbol{F}_{X}((a, a+T))\right) \\
& \quad=E\left(\mathscr{X}(a+T) \mid \partial \boldsymbol{F}_{X}(a)\right)+\int_{0}^{T} e^{(T-s) A}\left(P(s) \boldsymbol{e}^{*}+\sqrt{2 \pi^{-1}} \boldsymbol{b}\right) d \nu_{a}^{+}(s) \\
& \quad=e^{T A} J \cdot\left(X_{n_{0}}(a) \cdots X_{N-1}(a)\right)^{*}+\int_{0}^{T} e^{(T-s) A}\left(P(s) e^{*}+{\left.\sqrt{2 \pi^{-1}} \boldsymbol{b}\right) d \nu_{a}^{+}(s),}^{T^{*}},\right.
\end{aligned}
$$

where $J$ is the $N \times\left(N-n_{0}\right)$-matrix given by (3.4) and $P(t)$ is the unique solution of the following matrix Riccati equation: 


$$
\left\{\begin{aligned}
& \frac{d P(t)}{d t}=\left(A-\sqrt{2 \pi}^{-1} \boldsymbol{b} \cdot \boldsymbol{e}\right) P(t)+P(t)(A-\sqrt{2 \pi}-1 \boldsymbol{b} \cdot \boldsymbol{e})^{*} \\
&-P(t) \boldsymbol{e}^{*} \cdot \boldsymbol{e} \cdot P(t) \quad(t>0), \\
& P(0)=K_{x}(0)-J \cdot\left(K_{x}(0)_{m n}\right)_{n_{0} \leq m, n \leq N-1} J^{*} .
\end{aligned}\right.
$$

[3.2] In this subsection we shall obtain integral representations of the predictors $E\left(\mathscr{Y}(a-T) \mid F_{a}^{-(T)}\right)(a \in R, T \geq 0)$. Similarly as in Lemmas 3.1 and 3.2, we see from Theorems 2.3 and 2.4 that

LEMma 3.6. For any $a \in R$ and any $T \in(0, \infty)$,

$$
\begin{aligned}
& E\left(\mathscr{Y}(a-T) \mid F_{X}((a-T, a))\right) \\
& \quad=E\left(\mathscr{Y}(a-T) \mid \partial F_{X}(a)\right)+\int_{0}^{T} e^{(T-s) A}\left(Q_{a}(s) e^{*}+{\left.\sqrt{2 \pi^{-1}} b\right) d \nu_{a}^{-}(s),}\right.
\end{aligned}
$$

where $Q_{a}(t)=E\left\{\left(\mathscr{Y}(a-t)-E\left(\mathscr{Y}(a-t) \mid F_{a}^{-}(t)\right)\right)\right\} \cdot(\mathscr{Y}(a-t)-E(\mathscr{Y}(a-$ t) $\left.\left.\left.\mid \boldsymbol{F}_{a}^{-(}(t)\right)\right)^{*}\right\}$.

In the same way as Lemma 3.3, by Theorems 2.3, 2.4 and Lemma 3.6, we have

LEMMA 3.7. For any $a \in R, Q_{a}(t)$ satisfies the following matrix Riccati equation

$$
\left\{\begin{array}{l}
\frac{d Q_{a}(t)}{d t}=\left(A-\sqrt{2 \pi^{-1}} \boldsymbol{b} \cdot \boldsymbol{e}\right) Q_{a}(t)+Q_{a}(t)\left(A-\sqrt{2 \pi^{-1}} \boldsymbol{b} \cdot \boldsymbol{e}\right)^{*}-Q_{a}(t) \cdot \boldsymbol{e}^{*} \cdot \boldsymbol{e} \cdot Q_{a}(t), \\
Q_{a}(0)=K_{\mathscr{y}}(0)-\Pi_{a}(0),
\end{array}\right.
$$

where $\Pi_{a}(0)=E\left\{E\left(\mathscr{Y}(a) \mid \partial F_{X}(a)\right) \cdot E\left(\mathscr{Y}(a) \mid \partial F_{X}(a)\right)^{*}\right\}$.

Now we shall show

LEMMA 3.8. For any $a \in R \quad Q_{a}(0)=P(0)$.

Proof. Similarly as in Lemma 3.4, we see that

$$
\Pi_{a}(0)=\tilde{J} \cdot\left(K_{\vartheta}(0)_{m n}\right)_{n_{0} \leq m, n \leq N-1} \cdot \tilde{J}^{*},
$$

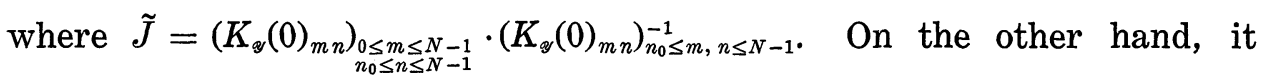
follows from (2.6) and (2.11) that $K_{y}(0)=K_{x x}(0)$. This implies that $Q_{a}(0)=P(0)$.

(Q.E.D.)

Therefore, we find from Lemmas 3.6, 3.7 and 3.8 that

THEOREM 3.2. For any $a \in R$ and any $T \in(0, \infty)$, 


$$
\begin{aligned}
& E\left(\mathscr{Y}(a-T) \mid \boldsymbol{F}_{X}((a-T, a))\right) \\
& \quad=E\left(\mathscr{Y}(a-T) \mid \partial \boldsymbol{F}_{X}(a)\right)+\int_{0}^{T} e^{(T-s) A}\left(P(s) \boldsymbol{e}^{*}+\sqrt{2 \pi^{-1}} \boldsymbol{b}\right) d \nu_{a}^{-}(s) \\
& \quad=e^{T A} J \cdot\left(Y_{n_{0}}(a) \cdots Y_{N-1}(a)\right)^{*}+\int_{0}^{T} e^{(T-s) A}\left(P(s) \boldsymbol{e}^{*}+\sqrt{2 \pi^{-1}} \boldsymbol{b}\right) d \nu_{a}^{-}(s),
\end{aligned}
$$

where $J$ is the $N \times\left(N-n_{0}\right)$-matrix given by (3.4) and $P(t)$ is the unique solution of the matrix Riccati equation (3.5).

[3.3] As an application of Theorems 3.1 and 3.2, we shall show

Theorem 3.3. For any $a \in R, t \in(0, \infty)$ and $T \in(0, \infty)$,

(i) $E\left(X(a+T+t) \mid F_{X}((a, a+T))\right)$

$$
\begin{aligned}
= & E\left(X(a+T+t) \mid \partial F_{X}(a)\right) \\
& +\left(0 \cdots 0\left(-c_{N}\right)^{-1} 2 \pi\right) \cdot \int_{0}^{T} f(T+t, s) d \nu_{a}^{+}(s)
\end{aligned}
$$

(ii) $E\left(X(a-T-t) \mid F_{X}((a-T, a))\right)$

$$
\begin{aligned}
= & E\left(X(a-T-t) \mid \partial F_{X}(a)\right) \\
& +\left(0 \cdots 0\left(-c_{N}\right)^{-1} 2 \pi\right) \cdot \int_{0}^{T} f(T+t, s) d \nu_{a}^{-}(s),
\end{aligned}
$$

where $\boldsymbol{f}(t, s)$ is the $N \times 1$-vector function for $a=0$ in (3.2).

Proof. By Theorems 2.1 (xi) and 3.1, we have

$$
\begin{aligned}
E\left(\mathscr{X}(a+T+t) \mid \boldsymbol{F}_{X}((a, a+T))\right) & \\
& =E\left(e^{t A} \mathscr{X}(a+T) \mid F_{X}((a, a+T))\right) \\
& =e^{t A}\left(E\left(\mathscr{X}(a+T) \mid \partial F_{X}(a)\right)+\int_{0}^{T} f(T, s) d \nu_{a}^{+}(s)\right) \\
& =E\left(\mathscr{X}(a+T+t) \mid \partial F_{X}(a)\right)+\int_{0}^{T} f(T+t, s) d \nu_{a}^{+}(s) .
\end{aligned}
$$

Therefore we obtain (i) noting Theorem 2.1 (i). By Theorem 2.3 (i) (xi) and Theorem 3.2, (ii) is similarly proved.

Immediately from Theorem 3.3, we have

THEOREM 3.4. For any $a \in R$ and $t \in(0, \infty)$,

(i) $X(a+t)=E\left(X(a+t) \mid \partial F_{X}(a)\right)$

$$
+\left(0 \cdots 0\left(-c_{N}\right)^{-1} 2 \pi\right) \cdot \int_{0}^{t} f(t, s) d \nu_{a}^{+}(s),
$$

(ii) $X(a-t)=E\left(X(a-t) \mid \partial F_{X}(a)\right)$

$$
+\left(0 \cdots 0\left(-c_{N}\right)^{-12 \pi}\right) \cdot \int_{0}^{t} f(t, s) d \nu_{a}^{-}(s) \text {. }
$$


We define two Gaussian processes $\boldsymbol{Y}_{ \pm}=\left(Y_{ \pm}(t) ; t \geq 0\right)$ by

$$
Y_{ \pm}(t)=X( \pm t)-E\left(X( \pm t) \mid \partial F_{X}(0)\right) .
$$

From Theorem 3.4, we have the following representations

$$
Y_{ \pm}(t)=\left(0 \cdots 0\left(-c_{N}\right)^{-1} 2 \pi\right) \cdot \int_{0}^{t} f(t, s) d \nu_{0}^{ \pm}(s) .
$$

It is easy to see from Theorems 2.2 (ii) and 2.4 (ii) that $\sigma\left(Y_{ \pm}(s) ; 0 \leq s\right.$ $\leq t) \vee \partial \boldsymbol{F}_{X}(0)=\sigma\left(\nu_{0}^{ \pm}(s) ; 0 \leq s \leq t\right) \vee \partial \boldsymbol{F}_{X}(0)$. Moreover $\boldsymbol{Y}_{ \pm}$and $\nu_{0}^{ \pm}$are independent of $\partial F_{X}(0)$. Therefore we obtain

$$
\sigma\left(Y_{ \pm}(s) ; 0 \leq s \leq t\right)=\sigma\left(\nu_{0}^{ \pm}(s) ; 0 \leq s \leq t\right) \quad(t \geq 0) .
$$

This implies that representations (3.7) are canonical ([3]).

\section{§4. Integral representations of the predictors (II)}

In the previous section we have obtained integral representations of $E\left(X(b+t) \mid F_{X}((a, b))\right)-E\left(X(b+t) \mid \partial F_{X}(a)\right)(a<b, t>0)$. The aim of this section is to obtain integral representations of $E\left(X(b+t) \mid F_{X}((a, b))\right)$ $E\left(X(b+t) \mid \partial F_{X}(b)\right)(a<b, t>0)$.

For any $a \in \boldsymbol{R}$ we define $N \times 1$-vectors $\boldsymbol{g}_{a}(t, s)(0 \leq s, t<\infty)$ by

$$
\boldsymbol{g}_{a}(t, s)=E\left\{\mathscr{Y}(a+t) \cdot\left(\mathscr{Y}(a-s)-E\left(\mathscr{Y}(a-s) \mid \boldsymbol{F}_{a}^{-}(s)\right)\right) *\right\} \cdot e^{*} .
$$

Then we shall prove

LEMMA 4.1. $\boldsymbol{g}_{a}(t, s)=(\partial / \partial s) E\left(\nu_{a}^{-}(s) \mathscr{Y}(a+t)\right)$.

Proof. We put $\widetilde{\mathscr{Y}}(s)=\mathscr{Y}(a-s)-E\left(\mathscr{Y}(a-s) \mid F_{a}^{-}(s)\right)$. It then follows from Theorems 2.3 (vi) and 2.4 (iv) that

$$
\nu_{a}^{-}(t)=e \cdot \int_{0}^{t} \widetilde{Y}(s) d s+B_{-}(a)-B_{-}(a-t) \quad(t \geq 0) .
$$

Therefore, by (2.10) and Theorem 2.3 (v) (vii), we have

$$
\begin{aligned}
E\left(\nu_{a}^{-}(s)\right. & \mathscr{Y}(a+t)) \\
= & e^{-t A} E\left\{\nu_{a}^{-}(s)\left(\mathscr{Y}(a)-\sqrt{2 \pi^{-1}} \int_{a}^{a+t} e^{(u-a) A} b d B_{-}(u)\right)\right\} \\
= & e^{-t A} E\left\{e \cdot \int_{0}^{s} \widetilde{\mathscr{Y}}(\iota) d \iota \cdot\left(\mathscr{Y}(a)-\sqrt{2 \pi^{-1}} \cdot \int_{a}^{a+t}\left(e^{(u-a) A} \boldsymbol{b} \cdot d B_{-}(u)\right)\right\}\right. \\
= & E\left(e \cdot \int_{0}^{s} \widetilde{\mathscr{Y}}(\iota) d_{\iota} \cdot \mathscr{Y}(a+t)\right) .
\end{aligned}
$$

This implies Lemma 4.1.

(Q.E.D.) 
Similarly as in Lemma 3.2, we can see from Theorem 2.4 and Lemma 4.1 that

LEMMA 4.2. For any $a \in R, t \in(0, \infty)$ and $T \in(0, \infty)$,

$$
E\left(\mathscr{Y}(a+t) \mid \boldsymbol{F}_{X}((a-T, a))\right)=E\left(\mathscr{Y}(a+t) \mid \partial \boldsymbol{F}_{X}(a)\right)+\int_{0}^{T} \boldsymbol{g}_{a}(t, s) d \nu_{a}^{-}(s) .
$$

Next we shall obtain an explicit form of $\boldsymbol{g}_{a}(t, s)$. We define for any $t \in[0, \infty)$ an $N \times N$-matrix $R(t)$ by

$$
R(t)=E\left\{\mathscr{Y}(t) \cdot\left(\mathscr{Y}(0)-E\left(\mathscr{Y}(0) \mid \partial F_{X}(0)\right)\right)^{*}\right\} .
$$

LEMMA 4.3. For any $a \in \boldsymbol{R}$

$$
\boldsymbol{g}_{a}(t, s)=R(t) \Phi^{*}(s) \boldsymbol{e}^{*} \quad(0 \leq s, t<\infty),
$$

where $\Phi(s)$ is the unique solution of the following linear differential equation

$$
\left\{\begin{array}{l}
\frac{d \Phi(s)}{d s}=\left(A-P(s) e^{*} \boldsymbol{e}-\sqrt{2 \pi}^{-1} \boldsymbol{b} \cdot \boldsymbol{e}\right) \Phi(s) \quad(s>0), \\
\Phi(0)=I_{N} .
\end{array}\right.
$$

In particular, $\boldsymbol{g}_{a}(t, s)$ are independent of $a$.

Proof. We put $R_{a}(t, s)=E\left\{\mathscr{Y}(a+t) \cdot\left(\mathscr{Y}(a-s)-E\left(\mathscr{Y}(a-s) \mid F_{a}^{-}(s)\right)\right)^{*}\right\}$. By Theorem 3.2,

$$
\begin{aligned}
R_{a}(t, s)= & E\left\{\mathscr{Y}(a+t)\left(\mathscr{Y}(a-s)-E\left(\mathscr{Y}(a-s) \mid \partial F_{X}(a)\right)\right)^{*}\right\} \\
& -E\left(\mathscr{Y}(a+t) \int_{0}^{s} f^{*}(s, \iota) d \nu_{a}^{-(\iota))}\right. \\
= & I-I I .
\end{aligned}
$$

It is easy to see from Theorem 2.4 and Lemma 4.2 that $I I=\int_{0}^{s} g_{a}(t, \iota) f^{*}(s, \iota) d \iota$. On the other hand, by Theorem 2.3 (iii) (ix), $I=E\{\mathscr{Y}(a+t) \cdot(\mathscr{Y}(a)-$ $\left.E\left(\mathscr{Y}(a) \mid \partial F_{X}(a)\right)^{*}\right\} \cdot e^{s A^{*}}$. Since $K_{\otimes}(0)=K_{x}(0)$ and $\partial F_{X}(a)=\sigma\left(Y_{n}(a) ; n_{0} \leq n\right.$ $\leq N-1)$, it can be seen that $E\left(\mathscr{Y}(a) \mid \partial F_{X}(a)\right)=J \cdot\left(Y_{n_{0}}(\alpha) \cdots Y_{N-1}(a)\right)^{*}$, where $J$ is the $N \times\left(N-n_{0}\right)$-matrix in (3.4). Therefore we find that $I=E\left\{\mathscr{Y}(t)\left(\mathscr{Y}(0)-E\left(\mathscr{Y}(0) \mid \partial F_{X}(0)\right) *\right\} e^{s A^{*}}=R(t) e^{s A^{*}}\right.$. Consequently, we have

$$
R_{a}(t, s)=R(t) e^{s A^{*}}-\int_{0}^{s} R_{a}(t, \iota) \boldsymbol{e}^{*} \cdot f^{*}(s, \iota) d \iota .
$$

Since $(\partial / \partial s) f^{*}(s, \iota)=f^{*}(s, \iota) A^{*}$ by $(3.2)$, we obtain the following linear 
differential equation

$$
\left\{\begin{array}{l}
\frac{\partial}{\partial s} R_{a}(t, s)=R_{a}(t, s)\left(A-P(s) e^{*} e-\sqrt{2 \pi^{-1}} \boldsymbol{b} \cdot \boldsymbol{e}\right)^{*} \quad(s>0), \\
R_{a}(t, 0)=R(t) .
\end{array}\right.
$$

Thus, using the unique solution $\Phi(t)$ of equation (4.4), we find that $R_{a}(t, s)=R(t) \Phi^{*}(s)$ and this completes the proof of Lemma 4.3. (Q.E.D.)

In the proof of Lemma 4.3, we have shown

$$
R(t)=K_{\mathscr{\Psi}}(0) e^{t A^{*}}-E\left(\mathscr{Y}(t) \cdot\left(Y_{n_{0}}(0) \cdots Y_{N-1}(0)\right)\right) \cdot J^{*},
$$

where $J$ is the $N \times\left(N-n_{0}\right)$-matrix in (3.4). Furthermore we define for any $t \in R$ an $N \times\left(N-n_{0}\right)$-matrix $J(t)$ by

$$
J(t)=\left(K_{\mathscr{\vartheta}}(t)_{m n}\right)_{\substack{0 \leq m \leq N-1 \\ n_{0} \leq n \leq N-1}} \cdot\left(K_{\vartheta}(0)_{m n}\right)_{n_{0} \leq m, n \leq N-1}^{-1} \cdot
$$

Immediately from Lemmas 4.2 and 4.3, we have

THEOREM 4.1. For any $a \in R, t \in(0, \infty)$ and $T \in(0, \infty)$,

$$
\begin{aligned}
& E\left(\mathscr{Y}(a+t) \mid F_{X}((a-T, a))\right) \\
& \quad=J(t) \cdot\left(Y_{n_{0}}(a) \cdots Y_{N-1}(a)\right)^{*}+\int_{0}^{T} R(t) \Phi^{*}(s) e^{*} d \nu_{a}^{-}(s) .
\end{aligned}
$$

Since $K_{x}(-t)=K_{y}(t)(t \geq 0)$, it is easy to see from (4.3) and (4.5) that $E\left\{\mathscr{X}(-t)\left(\mathscr{X}(0)-E\left(\mathscr{X}(0) \mid \partial F_{\mathscr{x}}(0)\right)\right)^{*}\right\}=R(t)(t \geq 0)$. Therefore, similarly as in Theorem 4.1, we can show from Theorems 2.1, 2.2, 3.1 and (3.3) that

THEOREM 4.2. For any $a \in R, t \in(0, \infty)$ and $T \in(0, \infty)$,

$$
\begin{aligned}
& E\left(\mathscr{X}(a-t) \mid \boldsymbol{F}_{X}((a, a+T))\right) \\
& \quad=J(t)\left(X_{n_{0}}(a) \cdots X_{N-1}(a)\right)^{*}+\int_{0}^{T} R(t) \Phi^{*}(s) e^{*} d \nu_{a}^{+}(s) .
\end{aligned}
$$

For any $t \in[0, \infty)$ we define a $1 \times N$-vector $\boldsymbol{r}(t)$ by

$$
r(t)=E\left\{X(t) \cdot\left(\mathscr{Y}(0)-E\left(\mathscr{Y}(0) \mid \partial F_{X}(0)\right)\right)^{*}\right\} .
$$

Since $X(t)=(-2 \pi) c_{N}^{-1} X_{N-1}(t)=(-2 \pi) c_{N}^{-1} Y_{N-1}(t)$, it can be seen from Theorems 4.1 and 4.2 that

THEOREM 4.3. For any $a \in R, t \in(0, \infty)$ and $T \in(0, \infty)$,

(i) $E\left(X(a+t) \mid F_{X}((a-T, a))\right)$ 


$$
=E\left(X(a+t) \mid \partial \boldsymbol{F}_{X}(a)\right)+\int_{0}^{T} \boldsymbol{r}(t) \Phi^{*}(s) \boldsymbol{e}^{*} d \nu_{a}^{-}(s),
$$

(ii) $E\left(X(a-t) \mid \boldsymbol{F}_{X}((a, a+T))\right)$

$$
=E\left(X(a-t) \mid \partial F_{X}(a)\right)+\int_{0}^{T} r(t) \Phi^{*}(s) e^{*} d \nu_{a}^{+}(s) .
$$

More generally, we define for any $Y \in M_{X}$ two $1 \times N$-vectors $\boldsymbol{r}_{ \pm}(Y)$ by

$$
\boldsymbol{r}_{+}(Y)=E\left\{Y \cdot\left(\mathscr{X}(0)-E\left(\mathscr{X}(0) \mid \partial \boldsymbol{F}_{X}(0)\right)\right)^{*}\right\}
$$

and

$$
\boldsymbol{r}_{-}(Y)=E\left\{Y \cdot\left(\mathscr{Y}(0)-E\left(\mathscr{Y}(0) \mid \partial \boldsymbol{F}_{X}(0)\right)\right) *\right\} \text {. }
$$

Note that $\boldsymbol{r}(t)=\boldsymbol{r}_{+}(X(-t))=\boldsymbol{r}_{-}(X(t))(t \in[0, \infty))$. Using the unitary operator $U(-a)$ in (2.12), we can prove, by Theorem 4.3,

THEOREM 4.4. Let any $a \in \boldsymbol{R}$ and $T \in(0, \infty)$ be fixed.

(i) For any $Y \in M_{X}^{+}(a)$

$E\left(Y \mid \boldsymbol{F}_{X}((a-T, a))\right)=E\left(Y \mid \partial \boldsymbol{F}_{X}(a)\right)+\int_{0}^{T} \boldsymbol{r}_{-}(U(-a) Y) \Phi^{*}(s) \boldsymbol{e}^{*} d \nu_{a}^{-}(s)$.

(ii) For any $Y \in M_{\bar{X}}^{-}(a)$

$$
E\left(Y \mid \boldsymbol{F}_{X}((a, a+T))\right)=E\left(Y \mid \partial \boldsymbol{F}_{X}(a)\right)+\int_{0}^{T} \boldsymbol{r}_{+}(U(-a) Y) \Phi^{*}(s) \boldsymbol{e}^{*} d \nu_{a}^{+}(s)
$$

\section{§5. Integral representations of the predictors (III)}

In this section we shall obtain integral representations of $E(X(a$ $\left.+t) \mid F_{X}((-a, a))\right)-E\left(X(a+t) \mid \partial F_{X}(0)\right)(a>0, t>0)$. We define for any $t \in[0, \infty)$ two $N \times N$-matrices $S_{ \pm}(t)$ by

$$
S_{+}(t)=E\left(\mathscr{Y}(t) \cdot \mathscr{X}^{*}(0)\right) \cdot K_{\mathscr{x}}(0)^{-1}
$$

and

$$
S_{-}(t)=E\left(\mathscr{X}(-t) \cdot \mathscr{Y}^{*}(0)\right) \cdot K_{\mathscr{x}}(0)^{-1} \text {. }
$$

As an application of Theorem 4.1, we shall prove

THEOREM 5.1. For any $a \in(0, \infty)$ and $t \in(0, \infty)$,

$$
\begin{aligned}
& E\left(\mathscr{Y}(a+t) \mid \boldsymbol{F}_{X}((-a, a))\right) \\
& \quad=E\left(\mathscr{Y}(a+t) \mid \partial \boldsymbol{F}_{X}(0)\right)+\int_{0}^{a} S_{+}(t) e^{(a-s) A}\left(P(s) \boldsymbol{e}^{*}+\sqrt{2 \pi^{-1}} \boldsymbol{b}\right) d \nu_{\partial}^{+}(s)
\end{aligned}
$$




$$
+\int_{a}^{2 a} R(t) \Phi^{*}(s) e^{*} d \nu_{a}^{-}(s) .
$$

Proof. It is easy to see from Theorem 4.1 that

$$
E\left(\mathscr{Y}(a+t) \mid \boldsymbol{F}_{X}((-a, a))\right)=E\left(\mathscr{Y}(a+t) \mid \boldsymbol{F}_{X}((0, a))\right)+\int_{a}^{2 a} R(t) \Phi^{*}(s) \boldsymbol{e}^{*} d \nu_{a}^{-}(s) .
$$

By Theorems 2.1 (iii) (iv) and 2.3 (iii) (iv), we can show that

$$
E\left(\mathscr{Y}(a+t) \mid F_{\bar{X}}(a)\right)=S_{+}(t) \mathscr{X}(a) .
$$

Therefore, it follows from Theorem 3.1 that

$$
\begin{aligned}
E\left(\mathscr{Y}(a+t) \mid F_{X}((0, a))\right) & \\
= & S_{+}(t) \cdot\left(E\left(\mathscr{X}(a) \mid \partial F_{X}(0)\right)+\int_{0}^{a} f(a, s) d \nu_{0}^{+}(s)\right) \\
& =E\left(\mathscr{Y}(a+t) \mid \partial F_{X}(0)\right)+\int_{0}^{a} S_{+}(t) f(a, s) d \nu_{0}^{+}(s),
\end{aligned}
$$

where $\boldsymbol{f}(a, s)$ are $N \times 1$-vectors in (3.2). Thus we have proved Theorem 5.1.

(Q.E.D.)

Similarly, we find from Theorems 3.2 and 4.2 that

THEOREM 5.2. For any $a \in(0, \infty)$ and $t \in(0, \infty)$,

$$
\begin{aligned}
& E\left(\mathscr{X}(-a-t) \mid \boldsymbol{F}_{X}((-a, a))\right) \\
& =E\left(\mathscr{X}(-a-t) \mid \partial \boldsymbol{F}_{X}(0)\right)+\int_{0}^{a} S_{-}(t) e^{(a-s) A}\left(P(s) e^{*}+\sqrt{2 \pi^{-1}} b\right) d \nu_{0}^{-}(s) \\
& \quad+\int_{a}^{2 a} R(t) \Phi^{*}(s) e^{*} d \nu_{-a}^{+}(s) .
\end{aligned}
$$

Remark 5.1. By Theorems 2.2 (ii) (iii) and 2.4 (ii) (iii) we note that the decompositions in Theorems 5.1 and 5.2 are orthogonal.

For any $t \in[0, \infty)$ we define two $1 \times N$-vectors $S_{ \pm}(t)$ by

$$
S_{+}(t)=E\left(X(t) \cdot \mathscr{X}^{*}(0)\right) \cdot K_{x}(0)^{-1}
$$

and

$$
S_{-}(t)=E\left(X(-t) \cdot \mathscr{Y}^{*}(0)\right) \cdot K_{x}(0)^{-1} .
$$

Since $X(t)=(-2 \pi) c_{N}^{-1} X_{N-1}(t)=(-2 \pi) c_{N}^{-1} Y_{N-1}(t)$, we can show from Theorems 5.1 and 5.2 that

THEOREM 5.3. For any $a \in(0, \infty)$ and $t \in(0, \infty)$, 
(i) $E\left(X(a+t) \mid F_{X}((-a, a))\right)$

$$
\begin{aligned}
= & E\left(X(a+t) \mid \partial \boldsymbol{F}_{X}(0)\right)+\int_{0}^{a} S_{+}(t) e^{(a-s) A}\left(P(s) \boldsymbol{e}^{*}+\sqrt{2 \pi^{-1}} \boldsymbol{b}\right) d \nu_{0}^{+}(s) \\
& +\int_{a}^{2 a} \boldsymbol{r}(t) \Phi^{*}(s) \boldsymbol{e}^{*} d \nu_{a}^{-}(s), \\
\text { (ii) } \quad E\left(X(-a-t) \mid \boldsymbol{F}_{X}((-a, a))\right) & \\
= & E\left(X(-a-t) \mid \partial \boldsymbol{F}_{X}(0)\right) \\
& +\int_{0}^{a} S_{-}(t) e^{(a-s) A}\left(P(s) \boldsymbol{e}^{*}+\sqrt{\left.2 \pi^{-1} b\right) d \nu_{0}^{-}(s)}\right. \\
& +\int_{a}^{2 a} \boldsymbol{r}(t) \Phi^{*}(s) \boldsymbol{e}^{*} d \nu_{-a}^{+}(s) .
\end{aligned}
$$

More generally, we define for any $Y \in M_{X}$ two $1 \times N$-vectors $S_{ \pm}(Y)$ by

$$
S_{+}(Y)=E\left(Y \cdot \mathscr{X}^{*}(0)\right) \cdot K_{x}(0)^{-1}
$$

and

$$
\boldsymbol{S}_{-}(Y)=E\left(Y \cdot \mathscr{Y}^{*}(0)\right) \cdot K_{x}(0)^{-1} .
$$

Using the unitary operators $U( \pm a)$ in (2.12), we can generalize Theorem 5.3 as follows.

THEOREM 5.4. Let any $a \in(0, \infty)$ be fixed.

(i) For any $Y \in \boldsymbol{M}_{\boldsymbol{X}}^{+}(a)$

$$
\begin{aligned}
& E\left(Y \mid \boldsymbol{F}_{X}((-a, a))\right) \\
& =E\left(Y \mid \partial \boldsymbol{F}_{X}(0)\right)+\int_{0}^{a} \boldsymbol{S}_{+}(U(-a) Y) e^{(a-s) A}\left(P(s) \boldsymbol{e}^{*}+\sqrt{\left.2 \pi^{-1} \boldsymbol{b}\right)} d \nu_{0}^{+}(s)\right. \\
& \quad+\int_{a}^{2 a} \boldsymbol{r}_{-}(U(-a) Y) \Phi^{*}(s) \boldsymbol{e}^{*} d \nu_{a}^{-}(s) .
\end{aligned}
$$

(ii) For any $Y \in \boldsymbol{M}_{\bar{X}}^{-}(-a)$

$$
\begin{aligned}
E\left(Y \mid \boldsymbol{F}_{X}((-a, a))\right) & \\
= & E\left(Y \mid \partial \boldsymbol{F}_{X}(0)\right)+\int_{0}^{a} \boldsymbol{S}_{-}(U(a) Y) \cdot e^{(a-s) A}\left(P(s) \boldsymbol{e}^{*}+\sqrt{2 \pi^{-1}} \boldsymbol{b}\right) d \nu_{0}^{-}(s) \\
& \quad+\int_{a}^{2 a} \boldsymbol{r}_{+}(U(a) Y) \cdot \Phi^{*}(s) \boldsymbol{e}^{*} d \nu_{-a}^{+}(s) .
\end{aligned}
$$

Remark 5.2. We note that the decompositions in Theorems 5.3 and 5.4 are orthogonal. 


\section{§6. Prediction errors}

In [6] we have obtained the following commutative diagram

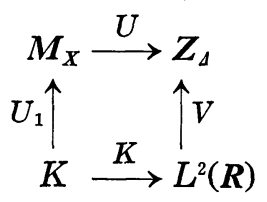

$$
U_{1}\left(k(\cdot-t)=X(t), \quad K(k(\cdot-t))=\sqrt{2 \pi^{-1}} E(t-\cdot) \quad \text { and } \quad U(X(t))=e^{i t \cdot} .\right.
$$

Similarly we have the following commutative diagram

$$
\begin{array}{cc}
M_{X} \stackrel{U}{\longrightarrow} Z_{\Delta} \\
\left.U_{1}\right|_{K} \stackrel{\check{K}}{\longrightarrow}{ }_{\check{V}}^{2}(R),
\end{array}
$$

$$
\check{K}(k(\cdot-t))=\sqrt{2 \pi^{-1}} E(\cdot-t)
$$

We note that

$$
f=\sqrt{2 \pi}^{-1}(V f \cdot \breve{h})^{\wedge}=\sqrt{2 \pi}^{-1}(\check{V} f \cdot h)^{\wedge} \quad\left(f \in L^{2}(R)\right) .
$$

Using $L^{2}$-functions $E_{n}$ in (2.4) we define $N$ functions $\varphi_{n}$ in $Z_{\Delta}(0 \leq n \leq$ $N-1)$ by

$$
\varphi_{n}=\sqrt{2 \pi}-1 V\left(\check{E}_{n}\right) .
$$

It is easy to see from (6.3) that

(6.5) $\quad V\left(\sqrt{2 \pi}^{-1} E_{n}(t-\cdot)\right)=e^{i t \cdot} \cdot \varphi_{n}$ and $\quad \check{V}\left(\sqrt{2 \pi}^{-1} E_{n}(\cdot-t)\right)=e^{i t \cdot} \cdot \check{\varphi}_{n}$.

Therefore it can be shown from (2.1), (2.6), (2.9) and (2.11) that

$$
U\left(X_{n}(t)\right)=e^{i t \cdot} \cdot \varphi_{n} \text { and } U\left(Y_{n}(t)\right)=e^{i t \cdot} \cdot \check{\varphi}_{n} \cdot
$$

Furthermore we are able to prove by Theorems 2.2 (iv) and 2.4 (iv) that

$$
U\left(\nu_{a}^{+}(t)\right)(\lambda)=\sqrt{2 \pi} \int_{a}^{a+t}\left\{b_{n_{0}}^{-1} \varphi_{n_{0}}(\lambda) i \lambda e^{i s \lambda}-P_{Z_{\Delta}((a, s))}(e \cdot U \mathscr{X}(s))(\lambda)\right\} d s
$$

and

$$
\begin{aligned}
U\left(\nu_{a}^{-}(t)\right)(\lambda)= & \sqrt{2 \pi} \int_{a-t}^{a}\left\{b_{n_{0}}^{-1} \varphi_{n_{0}}(-\lambda)(-i \lambda) e^{i s \lambda}\right. \\
& \left.-P_{Z_{\Delta}((s, a))}(\boldsymbol{e} \cdot U \mathscr{Y}(s))(\lambda)\right\} d s,
\end{aligned}
$$

where $U \mathscr{X}(s)=\left(U X_{0}(s), \cdots, U X_{N-1}(s)\right)^{*}$ and $U \mathscr{Y}(s)=\left(U Y_{0}(s), \cdots, U Y_{N-1}(s)\right)^{*}$. We define functions $D_{a}^{ \pm}(t, \lambda)$ by 


$$
\left\{\begin{array}{l}
D_{a}^{+}(t, \lambda)=\sqrt{2 \pi}\left\{b_{n_{0}}^{-1} \varphi_{n_{0}}(\lambda) \cdot i \lambda e^{i(t+a) \lambda}-P_{Z_{\Delta}((a, a+t))}(\boldsymbol{e} \cdot U \mathscr{X}(t+a))(\lambda)\right\}, \\
D_{a}^{-}(t, \lambda)=\sqrt{2 \pi}\left\{b_{n_{0}}^{-1} \varphi_{n_{0}}(-\lambda)(-i \lambda) \cdot e^{i(a-t) \lambda}-P_{Z_{\Delta}((a-t, a))}(\boldsymbol{e} \cdot U \mathscr{Y}(a-t))(\lambda)\right\} .
\end{array}\right.
$$

By (6.7), (6.8) and (6.9), it follows from Theorems 3.1 and 3.2 that

$$
\left\{\begin{array}{l}
D_{a}^{+}(t, \lambda)+\sqrt{2 \pi} \int_{0}^{t} \boldsymbol{e} \cdot \boldsymbol{f}(t, s) D_{a}^{+}(s, \lambda) d s \\
\quad=\sqrt{2 \pi}\left\{b_{n_{0}}^{-1} \varphi_{n_{0}}(\lambda) i \lambda e^{i(t+a) \lambda}-P_{\partial Z_{\Delta}(a)}(\boldsymbol{e} \cdot U \mathscr{X}(t+a))(\lambda)\right\} \\
D_{a}^{-}(t, \lambda)+\sqrt{2 \pi} \int_{0}^{t} \boldsymbol{e} \cdot \boldsymbol{f}(t, s) D_{a}^{-}(s, \lambda) d s \\
\quad=\sqrt{2 \pi}\left\{b_{n_{0}}^{-1} \varphi_{n_{0}}(-\lambda)(-i \lambda) e^{i(a-t) \lambda}-P_{\partial Z_{\Delta}(a)}(\boldsymbol{e} \cdot U \mathscr{Y}(a-t))(\lambda)\right\} .
\end{array}\right.
$$

In [6] we have introduced the function $P(\lambda, \varphi)(\lambda \in C, \varphi \in \mathcal{O}(\{0\}))$ defined by

$$
P(\lambda, \varphi)=(2 \pi)^{-1} \sum_{n=0}^{N-1}\left(\sum_{k=0}^{N-n-1} c_{n+k+1} \varphi^{(k)}(0)\right)(-i \lambda)^{n} .
$$

We then define $N$ functions $P_{n}(0 \leq n \leq N-1)$ by

$$
P_{n}(\lambda)=(n !)^{-1} P\left(\lambda, x^{n}\right) \text {. }
$$

Firstly we shall prove

LEMMA 6.1. $\varphi_{n}=P_{n}$ for any $n \in\left\{n_{0}, n_{0}+1, \cdots, N-1\right\}$.

Proof. We define $N$ real $L^{2}$-functions $F_{n}(0 \leq n \leq N-1)$ by

$$
F_{n}(t)=\sqrt{2 \pi^{-1}} \chi_{(0, \infty)}(t)\left(e^{t A}\left(\begin{array}{c}
1 \\
0 \\
\vdots \\
0
\end{array}\right)\right)_{n} .
$$

By Lemma 8.2 in [6] and (2.3), (6.8) in [7], we have

$$
F_{n}=(n !)^{-1}\left\{P\left(-\cdot, x^{n}\right) \cdot P(-\cdot)^{-1}\right\}^{\wedge}=\left(\check{P}_{n} \check{P}^{-1}\right)^{\wedge} \text {. }
$$

Since $P_{n}\left(n_{0} \leq n \leq N-1\right), P$ and $Q$ are polynomials of at most order $N-n_{0}-1, N$ and $n_{0}$, respectively, we see from (2.7) in [7] and Lemma 4.1 in [7] that

$$
E_{n}=\left(\check{P}_{n} \cdot \check{Q} \cdot \check{P}^{-1}\right)^{\wedge}=\left(\check{P}_{n} \cdot h\right)^{\wedge} \quad\left(n_{0} \leq n \leq N-1\right) .
$$

This implies Lemma 6.1 by (6.3) and (6.5).

Noting that $P_{N-1}=-(2 \pi)^{-1} c_{N}$, we note by (6.11), (6.12) and Lemma 6.1 that 


$$
b_{n_{0}}^{-1} \varphi_{n_{0}}(\lambda) i \lambda= \begin{cases}e \cdot\left(P_{0}(\lambda) \cdots P_{N-1}(\lambda)\right)^{*} & \text { if } n_{0} \geq 1, \\ \left(2 \pi b_{0}\right)^{-1} \sum_{k=1}^{N} c_{k}(-i \lambda)^{k} & \text { if } n_{0}=0 .\end{cases}
$$

Moreover, by Theorem 3.1 and (6.6),

$$
P_{\partial Z_{\Delta}(a)}(e \cdot U \mathscr{X}(t+a))(\lambda)=e^{i a \lambda} e \cdot e^{t A} J \cdot\left(\varphi_{n_{0}}(\lambda) \cdots \varphi_{N-1}(\lambda)\right)^{*} .
$$

Therefore, defining functions $\psi(t, \lambda)$ by

$$
\psi(t, \lambda)=\sqrt{2 \pi}\left\{b_{n_{0}}^{-1} e^{i t \lambda} \varphi_{n_{0}}(\lambda) i \lambda-e \cdot e^{t A} J \cdot\left(\varphi_{n_{0}}(\lambda) \cdots \varphi_{N-1}(\lambda)\right)^{*}\right\}
$$

we find from (6.10) that

$$
D_{a}^{+}(t, \lambda)+\sqrt{2 \pi} \int_{0}^{t} \boldsymbol{e} \cdot \boldsymbol{f}(t, s) D_{a}^{+}(s, \lambda) d s=e^{i a \lambda} \psi(t, \lambda) .
$$

By the uniqueness of solution of Volterra equation (6.19), we have

$$
D_{a}^{+}(t, \lambda)=e^{i a \lambda} D_{0}^{+}(t, \lambda) .
$$

Similarly, by Theorem 3.2, (6.6) and (6.10),

$$
D_{a}^{-}(t, \lambda)+\sqrt{2 \pi} \int_{0}^{t} \boldsymbol{e} \cdot \boldsymbol{f}(t, s) D_{a}^{-}(s, \lambda) d s=e^{i a \lambda} \psi(t,-\lambda)
$$

and

$$
D_{a}^{-}(t, \lambda)=e^{i a \lambda} D_{0}^{-}(t, \lambda) .
$$

In particular, we see from (6.19) and (6.21) that

$$
D_{0}^{+}(t, \lambda)=D_{0}^{-}(t,-\lambda) \text {. }
$$

Next we shall obtain explicit representations of functions $f(t, s)$ and $g(t, s)$ in (3.2) and (4.1), respectively.

LEMMA 6.2. (i) $f(t, s)=\left(D_{0}^{+}(s, \cdot), e^{i t \cdot} \cdot \varphi\right)_{\Delta}$,

(ii) $\boldsymbol{g}(t, s)=R(t) \Phi^{*}(s) \boldsymbol{e}^{*}=\left(D_{0}^{-}(s, \cdot), e^{i t \cdot} \cdot \check{\varphi}\right)_{\Delta}$, where $\varphi=\left(\varphi_{0} \cdots \varphi_{N-1}\right)^{*}$.

Proof. By (6.7), (6.8) and (6.9),

$$
U\left(\nu_{a}^{ \pm}(s)\right)(\lambda)= \pm \int_{a}^{a \pm s} D_{a}^{ \pm}( \pm(\iota-a), \lambda) d \iota .
$$

Therefore it follows from Lemma 3.1 and (6.6) that

$$
f(t, s)=\frac{\partial}{\partial s} E\left(\nu_{0}^{+}(s) \cdot \mathscr{X}(t)\right)=\left(D_{0}^{+}(s, \cdot), e^{i t \cdot} \cdot \varphi\right)_{\Delta} .
$$


Similarly we find from Lemmas $4.1,4.3$ and (6.6) that

$$
\boldsymbol{g}(t, s)=R(t) \Phi^{*}(s) \boldsymbol{e}^{*}=\frac{\partial}{\partial s} E\left(\nu_{0}^{-}(s) \mathscr{Y}(t)\right)=\left(D_{0}^{-}(s, \cdot), e^{i t \cdot} \cdot \check{\varphi}\right)_{\Delta} \cdot
$$

LEMMA 6.3. $\boldsymbol{S}_{ \pm}(t) \boldsymbol{f}(a, s)=\left(D_{0}^{+}(s, \cdot), e^{i(a+t) \cdot}\right)_{\Delta}$.

Proof. By (5.3), (5.4), Theorems 2.1 (ix) and 2.3 (ix),

$$
S_{ \pm}(t)=\left(0 \cdots 0\left(-c_{N}\right)^{-1} 2 \pi\right) e^{t A} .
$$

Therefore it follows from (3.2) and Lemma 6.2 that

$$
\begin{aligned}
\boldsymbol{S}_{ \pm}(t) \boldsymbol{f}(a, s) & =\left(0 \cdots 0\left(-c_{N}\right)^{-1} 2 \pi\right) \boldsymbol{f}(a+t, s) \\
& =\left(0 \cdots 0\left(-c_{N}\right)^{-1} 2 \pi\right)\left(D_{0}^{+}(s, \cdot), e^{i(a+t) \cdot} \cdot \varphi\right)_{\Delta} .
\end{aligned}
$$

Noting that $\varphi_{N-1}=P_{N-1}=-(2 \pi)^{-1} c_{N}$, we have Lemma 6.3.

(Q.E.D.)

LEMMA 6.4. $\boldsymbol{r}(t) \Phi^{*}(s) e^{*}=\left(D_{0}^{-}(s, \cdot), e^{i t \cdot}\right)_{\Delta}$.

Proof. By (4.3), (4.7) and Lemma 6.2,

$$
\begin{aligned}
\boldsymbol{r}(t) \Phi^{*}(s) \boldsymbol{e}^{*} & =\left(0 \cdots 0\left(-c_{N}\right)^{-1} 2 \pi\right) R(t) \Phi^{*}(s) \boldsymbol{e}^{*} \\
& =\left(0 \cdots 0\left(-c_{N}\right)^{-1} 2 \pi\right)\left(D_{0}^{-}(s, \cdot), e^{i t \cdot} \cdot \check{\varphi}\right)_{\Delta} \\
& =\left(D_{0}^{-}(s, \cdot), e^{i t \cdot}\right)_{\Delta} .
\end{aligned}
$$

Now we shall obtain explicit integral representations of prediction errors.

THEOREM 6.1. For any $a \in R, t \in(0, \infty)$ and $T \in(0, \infty)$,

$$
\begin{aligned}
& \left\|X(a+t)-E\left(X(a+t) \mid F_{X}((a-T, a))\right)\right\|^{2} \\
& \quad=\left\|X(a-t)-E\left(X(a-t) \mid F_{X}((a, a+T))\right)\right\|^{2} \\
& \quad=\int_{0}^{t}\left(D_{0}^{+}(s, \cdot), e^{i t \cdot}\right)_{\Delta}^{2} d s-\int_{0}^{T}\left(D_{0}^{-}(s, \cdot), e^{i t \cdot}\right)_{\Delta}^{2} d s .
\end{aligned}
$$

Proof. Using Lemmas 6.4 and 6.2, we see from Theorems 4.3 and 3.4 that

$$
\begin{aligned}
& \left\|X(a+t)-E\left(X(a+t) \mid \boldsymbol{F}_{X}((a-T, a))\right)\right\|^{2} \\
& \quad=\|X(a+t)\|^{2}-\left\|E\left(X(a+t) \mid \boldsymbol{F}_{X}((a-T, a))\right)\right\|^{2} \\
& \quad=\|X(a+t)\|^{2}-\left\|E\left(X(a+t) \mid \partial F_{X}(a)\right)\right\|^{2}-\int_{0}^{T}\left(D_{0}^{-}(s, \cdot), e^{i t \cdot}\right)_{\Delta}^{2} d s \\
& \quad=\left\|X(a+t)-E\left(X(a+t) \mid \partial F_{X}(a)\right)\right\|^{2}-\int_{0}^{T}\left(D_{0}^{-}(s, \cdot), e^{i t \cdot}\right)_{\Delta}^{2} d s
\end{aligned}
$$




$$
=\int_{0}^{t}\left(D_{0}^{+}(s, \cdot), e^{i t \cdot}\right)_{\Delta}^{2} d s-\int_{0}^{T}\left(D_{0}^{-}(s, \cdot), e^{i t \cdot}\right)_{\Delta}^{2} d s .
$$

The rest is similarly proved.

(Q.E.D.)

THEOREM 6.2. For any $a \in(0, \infty)$ and $t \in(0, \infty)$,

$$
\begin{aligned}
& \left\|X( \pm(a+t))-E\left(X\left( \pm(a+t) \mid F_{X}((-a, a))\right)\right)\right\|^{2} \\
& \quad=\int_{a}^{a+t}\left(D_{0}^{+}(s, \cdot), e^{i(a+t) \cdot}\right)_{\Delta}^{2} d s-\int_{a}^{2 a}\left(D_{0}^{-}(s, \cdot), e^{i t \cdot}\right)_{\Delta}^{2} d s .
\end{aligned}
$$

Proof. Using Lemmas 6.3, 6.4 and 6.2, we find from Theorems 5.3 and 3.4 that

$$
\begin{aligned}
& \left\|X( \pm(a+t))-E\left(X( \pm(a+t)) \mid \boldsymbol{F}_{X}((-a, a))\right)\right\|^{2} \\
& =\|X( \pm(a+t))\|^{2}-\left\|E\left(X( \pm(a+t)) \mid \boldsymbol{F}_{X}((-a, a))\right)\right\|^{2} \\
& =\|X( \pm(a+t))\|^{2}-\left\|E\left(X( \pm(a+t)) \mid \partial \boldsymbol{F}_{X}(0)\right)\right\|^{2} \\
& \quad-\int_{0}^{a}\left(D_{0}^{+}(s, \cdot), e^{i(a+t) \cdot}\right)_{\Delta}^{2} d s-\int_{a}^{2 a}\left(D_{0}^{-}(s, \cdot), e^{i t \cdot}\right)_{\Delta}^{2} d s \\
& =\left\|X( \pm(a+t))-E\left(X( \pm(a+t)) \mid \partial \boldsymbol{F}_{X}(0)\right)\right\|^{2} \\
& \quad-\int_{0}^{a}\left(D_{0}^{+}(s, \cdot), e^{i(a+t) \cdot}\right)_{\Delta}^{2} d s-\int_{a}^{2 a}\left(D_{0}^{-}(s, \cdot), e^{i t \cdot}\right)_{\Delta}^{2} d s \\
& =\int_{a}^{a+t}\left(D_{0}^{+}(s, \cdot), e^{i(a+t) \cdot}\right)_{\Delta}^{2} d s-\int_{a}^{2 a}\left(D_{0}^{-}(s, \cdot), e^{i t \cdot}\right)_{\Delta}^{2} d s .
\end{aligned}
$$

\section{§7. Integral representations of the predictors (IV)}

In this section we shall give explicit integral representations of prediction formulas in $\S 3, \S 4$ and $\S 5$ using the section of the previous section. By Theorem 3.3 and Lemma 6.2,

TheOREM 7.1. For any $a \in R, t \in(0, \infty)$ and $T \in(0, \infty)$,

(i) $E\left(X(a+T+t) \mid F_{X}((a, a+T))\right)$

$$
=E\left(X(a+T+t) \mid \partial F_{X}(a)\right)+\int_{0}^{T}\left(D_{0}^{+}(s, \cdot), e^{i(T+t) \cdot}\right){ }_{\Delta} d \nu_{a}^{+}(s)
$$

(ii) $E\left(X(a-t-T) \mid F_{X}((a-T, a))\right)$

$$
=E\left(X(a-T-t) \mid \partial \boldsymbol{F}_{X}(a)\right)+\int_{0}^{T}\left(D_{0}^{+}(s, \cdot), e^{i(T+t) \cdot}\right)_{\Delta} d \nu_{a}^{-}(s) .
$$

Similarly, we see from Theorem 3.4 and Lemma 6.2 that

THEOREM 7.2. For any $a \in \boldsymbol{R}$ and $t \in(0, \infty)$,

$$
X(a \pm t)=E\left(X(a \pm t) \mid \partial F_{X}(a)\right)+\int_{0}^{t}\left(D_{0}^{+}(s, \cdot), e^{i t \cdot}\right)_{\Delta} d \nu_{a}^{ \pm}(s) .
$$


By Theorem 4.3 and Lemma 6.4,

THEOREM 7.3. For any $a \in R, t \in(0, \infty)$ and $T \in(0, \infty)$,

(i) $E\left(X(a+t) \mid \boldsymbol{F}_{X}((a-T, a))\right)$

$$
=E\left(X(a+t) \mid \partial F_{X}(a)\right)+\int_{0}^{T}\left(D_{0}^{-}(s, \cdot), e^{i t \cdot}\right)_{\Delta} d \nu_{a}^{-}(s)
$$

(ii) $E\left(X(a-t) \mid F_{X}((a, a+T))\right)$

$$
=E\left(X(a-t) \mid \partial F_{X}(a)\right)+\int_{0}^{T}\left(D_{0}^{-}(s, \cdot), e^{i t \cdot}\right){ }_{\Delta} d \nu_{a}^{+}(s) .
$$

From Theorem 5.3, Lemmas 6.3 and 6.4, it follows that

THEOREM 7.4. For any $a \in(0, \infty)$ and $t \in(0, \infty)$,

$$
\begin{aligned}
E(X( \pm & \left.(a+t)) \mid F_{X}((-a, a))\right) \\
= & E\left(X( \pm(a+t)) \mid \partial F_{X}(0)\right)+\int_{0}^{a}\left(D_{0}^{+}(s, \cdot), e^{i(a+t) \cdot}\right)_{\Delta} d \nu_{0}^{ \pm}(s) \\
& \quad+\int_{a}^{2 a}\left(D_{0}^{-}(s, \cdot), e^{i t \cdot}\right)_{\Delta} d \nu_{ \pm}^{\mp}(s) .
\end{aligned}
$$

By (6.6), (6.20), (6.22) and (6.24), we have

(7.1) $\frac{\partial}{\partial s} E\left(\mathscr{X}(a+t) \nu_{a}^{-}(s)\right)=\frac{\partial}{\partial s} E\left(\mathscr{Y}(a-t) \nu_{a}^{+}(s)\right)=\left(D_{0}^{-}(s, \cdot), e^{i t \cdot} \varphi\right)_{\Delta}$.

Therefore, similarly as in Lemma 3.2, we obtain the following Theorem 7.5 as a supplement of Theorems 4.1 and 4.2 .

THEOREM 7.5. For any $a \in R, t \in(0, \infty)$ and $T \in(0, \infty)$,

(i) $E\left(\mathscr{X}(a+t) \mid \boldsymbol{F}_{\boldsymbol{X}}((a-T, a))\right.$

$$
=E\left(\mathscr{X}(a+t) \mid \partial F_{X}(a)\right)+\int_{0}^{T}\left(D_{0}^{-}(s, \cdot), e^{i t \cdot} \varphi\right)_{\Delta} d \nu_{a}^{-}(s),
$$

(ii) $E\left(\mathscr{Y}(a-t) \mid F_{X}((a, a+T))\right)$

$$
=E\left(\mathscr{Y}(a-t) \mid \partial F_{X}(a)\right)+\int_{0}^{T}\left(D_{0}^{-}(s, \cdot), e^{i t} \cdot \varphi\right)_{\Delta} d \nu_{a}^{+}(s) .
$$

Furthermore, as a supplement of Theorems 5.1 and 5.2, we shall prove

THEOREM 7.6. For any $a \in(0, \infty)$ and $t \in(0, \infty)$,

(i) $E\left(\mathscr{X}(a+t) \mid \boldsymbol{F}_{X}((-a, a))\right)$

$$
\begin{aligned}
= & E\left(\mathscr{X}(a+t) \mid \partial F_{X}(0)\right)+\int_{0}^{a}\left(D_{0}^{+}(s, \cdot), e^{i(a+t)} \cdot \varphi\right)_{\Delta} d \nu_{0}^{+}(s) \\
& +\int_{a}^{2 a}\left(D_{0}^{-}(s, \cdot), e^{i t \cdot} \varphi\right)_{\Delta} d \nu_{a}^{-}(s),
\end{aligned}
$$

(ii) $E\left(\mathscr{Y}(-a-t) \mid F_{X}((-a, a))\right)$ 


$$
\begin{aligned}
= & E\left(\mathscr{Y}(-a-t) \mid \partial F_{X}(0)\right)+\int_{0}^{a}\left(D_{0}^{+}(s, \cdot), e^{i(a+t) \cdot} \varphi\right)_{\Delta} d \nu_{0}^{-}(s) \\
& +\int_{a}^{2 a}\left(D_{0}^{-}(s, \cdot), e^{i t \cdot} \varphi\right)_{\Delta} d \nu_{-a}^{+}(s) .
\end{aligned}
$$

Proof. By Theorems 7.5 (i), 2.1 (ix), 3.1 and Lemma 6.2,

$$
\begin{aligned}
E\left(\mathscr{X}(a+t) \mid \boldsymbol{F}_{X}((-a, a))\right) \\
\quad=E\left(\mathscr{X}(a+t) \mid \partial \boldsymbol{F}_{X}(a)\right)+\int_{0}^{2 a}\left(D_{0}^{-}(s, \cdot), e^{i t \cdot \varphi)_{\Delta} d \nu_{a}^{-}(s)}\right. \\
=E\left(\mathscr{X}(a+t) \mid F_{X}((0, a))+\int_{a}^{2 a}\left(D_{0}^{-}(s, \cdot), e^{i t \cdot} \varphi\right)_{\Delta} d \nu_{a}^{-}(s)\right. \\
=E\left(\mathscr{X}(a+t) \mid \partial F_{X}(0)\right)+\int_{0}^{a}\left(D_{0}^{-}(s, \cdot), e^{i(a+t) \cdot} \varphi\right)_{\Delta} d \nu_{0}^{+}(s) .
\end{aligned}
$$

Similarly, we have (ii) from Theorems 7.5 (ii), 2.3 (ix), 3.2 and Lemma 6.2 .

(Q.E.D.)

Remark 7.1. Theorem 7.3 (resp. Theorem 7.4) follows immediately from Theorem 7.5 (resp. Theorem 7.6).

Remark 7.2. The decompositions in Theorems 7.4 and 7.6 are orthogonal.

\section{REFERENCES}

[1] H. Dym and H. P. McKean, Jr.: Application of de Branges spaces of integral functions to the prediction of stationary Gaussian processes, Illinois J. Math. 45 (1970), 299-343.

[2] - Gaussian processes, Function Theory, and the Inverse Spectral Problem, Academic Press, New York, 1976.

[ 3 ] T. Hida: Canonical representations of Gaussian processes and their applications, Mem. Coll. Sci. Univ. Kyoto, Ser. A, Math. 34 (1960), 109-155.

[4] T. Kailath: A note on least squares estimation by the innovation method, SIAM J. Control 10 (1972), 477-486.

[5] G. Kallianpur, M. Fujisaki and H. Kunita: Stochastic differential equations for the nonlinear filtering problem, Osaka J. Math. 9 (1972), 19-40.

[6] Y. Okabe: Stationary Gaussian processes with Markovian property and M. Sato's hyperfunctions, Japanese J. of Math. 41 (1973), 69-122.

[ 7] - On the structure of splitting fields of stationary Gaussian processes with finite multiple Markovian property, Nagoya Math. J. 54 (1974), 191-213.

Department of Mathematics

Faculty of Science

University of Tokyo

Hongo, Tokyo, Japan 